\title{
Critiques envers les recommandations de la CFPS concernant l'infectiosité des personnes séropositives sous TAR
}

\section{Sida Information Suisse *}

* Dr Kurt April, Horgen; Dr Alessandro Caponi, Lausanne; Pr Marcel Capt, Les Charbonnières; Dr Giovanni Fantacci, Niederhasli, Pr Max Geiser, Wabern; Dr Ralph Kaiser, Zurich Dr Thomas Keller, Pfäffikon SZ Pr Hans Koblet, Burgdorf; Dr Eric Rochat, Saint-Légier Ilona Singer, juriste, Zurich; Dr Wolfgang Steinke, Schaffhouse; Dr Dieter Walch, Vaduz

Réponse de la CFPS dès la page 1304.
Il est certes réjouissant d'apprendre que les personnes séropositives sous traitement antirétroviral (TAR) sont nettement moins contagieuses. Néanmoins, Sida Information Suisse (SIS) considère qu'au vu des faits scientifiques actuels, il est irresponsable de partir du principe que les personnes séropositives sous TAR ne sont pas contagieuses. En effet, même si le risque de contamination est faible, il ne peut être jugé négligeable. SIS est donc opposée à la modification de la jurisprudence selon laquelle une personne séropositive sous traitement antirétroviral qui ne respecterait pas les règles de prévention ne serait pas passible de sanctions. Cette mesure aurait un effet contre-productif sur la prévention de l'infection à VIH. En outre, les recommandations de la Commission fédérale pour les questions liées au sida (CFPS) constituent un mauvais signal envoyé à la population car elles pourraient lui faire croire que l'infection à VIH ne présente plus guère de danger. SIS se rallie donc aux critiques émises par les experts du monde entier envers les recommandations précitées. Il serait inapproprié que la Suisse fasse cavalier seul dans ce domaine.

\section{Le VIH, virus rusé}

On connaît l'infection à VIH depuis 25 ans. Pendant cette période, des sommes extrêmement élevées ont été investies dans la recherche, le traitement et la prévention pour lutter contre cette maladie infectieuse à l'issue presque toujours fatale. Le lentivirus IH est plus rusé que d'autres virus ou bactéries et nous sommes encore loin de la mise au point d'un vaccin. D'après les estimations de l'OMS [1], le nombre des personnes contaminées ne cesse d'augmenter dans le monde, y compris en Europe et en Amérique du Nord.

Un des plus grands succès remportés jusqu'ici dans la lutte contre l'épidémie a été l'introduction des thérapies antirétrovirales (TAR) qui ont transformé une infection à issue fatale en une infection chronique. Cela a permis de baisser nettement les taux de cas de sida et de décès par sida dans les pays occidentaux. L'espérance et la qualité de vie des personnes contaminées ont pu être nettement améliorées.
Toutefois, l'infection à VIH reste incurable. Certes, les TAR permettent de réduire la concentration de virus dans le sang (charge virale) en dessous du seuil de détection mais quelques jours après l'arrêt du traitement, on observe une augmentation rapide de cette virémie [2].

\section{Les TAR réduisent l'infectiosité}

La charge virale dans les sécrétions génitales se comporte comme la charge virale dans le sang. C'est pourquoi les TAR réduisent considérablement l'infectiosité de toutes les voies de contamination (sexuelle, de la mère à l'enfant, par le sang). Dans certaines études portant sur des couples sérodifférents (un seul des partenaires est contaminé) dont le partenaire contaminé était sous TAR, on n'a pas constaté de transmission du VIH [3].

\section{Prise de position de la CFPS} sur l'infectiosité et la prévention du VIH La CFPS prétend dans sa prise de position [4] que l'infectiosité des personnes séropositives suivant un TAR ayant complètement supprimé la virémie est si faible que l'on peut négliger le risque de contamination. Elles ne peuvent plus transmettre le VIH par voie sexuelle ou par voie maternofœtale. En conséquence, ces personnes peuvent renoncer aux mesures de prévention telles que l'usage du préservatif et ne sont plus punissables en cas de rapports sexuels non protégés.

La CFPS propose de limiter l'abandon de l'usage du préservatif aux couples sérodifférents lorsque «la personne séropositive sous TAR efficace» répond aux caractéristiques suivantes: 1. Elle «applique le traitement rétroviral à la lettre» et est suivie par un médecin. 2. Sa charge virale se situe en dessous du seuil de détection depuis au moins 6 mois. 3. Elle n'est atteinte d'aucune autre MST.

\section{Large écho médiatique et critiques de spécialistes dans le monde entier}

La prise de position de la CFPS a suscité un large écho médiatique. On a pu avoir l'impression que toutes les personnes contaminées étaient traitées 
efficacement, qu'elles jouissaient d'une qualité et d'une espérance de vie normales et qu'elles n'étaient plus contagieuses, bref que la CFPS avait donné le signal de fin d'alerte quant aux dangers de l'épidémie.

Un exemple de cette manière de comprendre le message nous a été fourni par la Neue Zürcher Zeitung - journal aux jugements généralement équilibrés et critiques - dans un article intitulé «A nouvelle situation, nouvelle jurisprudence»: L'auteur demande une nouvelle jurisprudence pour toutes les personnes séropositives qui suivent un traitement. En outre, il évoque l'opportunité de renoncer à condamner «les séropositifs qui se soumettent à un traitement, n'informent pas leur partenaire de leur statut et ne se protègent pas.» [5]

De nombreuses instances sanitaires, comme l'OMS [6], les Centers of Desease Control (CDC) américains [7], la Commission de la santé de l'Union européenne et le Conseil national du sida (CNS) français [8] prennent leurs distances à l'égard de la CFPS et mettent en garde: on ne doit pas, à partir d'études comme celles mentionnées par la CFPS, faire croire que le sida est devenu une maladie moins grave. Elles insistent sur le fait que l'on ne peut pas garantir à 100\% que les patients traités de manière efficace ne sont pas contagieux, si bien qu'il n'est pas indiqué de modifier les mesures de prévention. Toutes les personnes séropositives sexuellement actives doivent utiliser - et cela correctement - un préservatif lors de chaque rapport sexuel.

\section{Contagiosité et taux de transmission du VIH sous TAR}

Actuellement, il existe encore de nombreuses questions ouvertes, des résultats d'études peu clairs ou contradictoires qui incitent à être prudent en matière de contagiosité.

1. Plusieurs études ont constaté la présence d'ADN de virus associés aux cellules dans les sécrétions génitales de certaines personnes séropositives sous TAR efficace et dont la charge virale se situait en dessous du seuil de détection $[9,10]$.

2. Si un patient sous TAR efficace contracte une autre MST, la virémie augmente dans les sécrétions génitales, mais moins qu'en l'absence de TAR efficace $[11,12]$. C'est aussi possible lors d'autres processus inflammatoires des organes génitaux. Ces résultats étayent également l'hypothèse selon laquelle les ADN associés aux cellules que l'on observe aussi sous TAR pourraient être infectieux.

3. Les TAR peuvent empêcher la transmission de la mère à l'enfant $[13,14]$ mais ce n'est pas toujours le cas, contrairement à ce que laisse entendre la CFPS. Certaines études ont constaté quelques transmissions du VIH malgré un TAR efficace [15]. On suppose qu'elles sont dues à des épisodes passagers de réplication virale et à des microtraumatismes dans le placenta [16].

4. En raison de la variabilité du VIH, la propagation mondiale des TAR entraîne inévitablement une transmission accrue de virus résistants aux médicaments [17].

On a trop souvent déjà sous-estimé les ruses du virus IH, si bien que SIS recommande de prendre au sérieux les problèmes soulevés ci-dessus et de ne pas faire croire qu'il n'existe plus de danger de transmission du VIH.

A partir des mêmes résultats, d'autres chercheurs tirent d'autres conclusions que la CFPS. Ainsi Neely et al. [9] insistent sur la possibilité, même en cas de TAR efficace, d'une transmission de la mère à l'enfant ou d'un partenaire sexuel à l'autre ainsi que sur la possibilité de la propagation de virus résistants. Aussi demandent-ils de conseiller les personnes séropositives en conséquence.

\section{Conseil médical: recommandations contre-productives de la CFPS}

SIS juge indispensable d'informer les personnes séropositives sous ART et leur partenaire sur les risques de transmission du VIH. Même les faibles risques doivent être abordés. Aussi critique-t-elle la recommandation de la CFPS aux médecins traitants d'informer les personnes séropositives sous TAR efficace qu'elles ne sont pas contagieuses, indépendamment du fait qu'elles vivent ou non une relation fidèle. La CFPS justifie cette recommandation en disant qu'elle «peut signifier un soulagement» car la peur de contaminer le/la partenaire peut compromettre la vie sexuelle des personnes séropositives.

Il est vrai que la peur naturelle de contaminer le/la partenaire, de même que la crainte d'être condamné pour transmission du VIH peuvent conduire à des troubles de la sexualité $[18,19]$. Cependant une crainte justifiée n'est-elle pas de bon conseil? Une diminution du désir sexuel n'est-elle pas l'expression du respect d'autrui? De nombreuses personnes séropositives - et précisément à la suite de leur expérience de l'infection à VIH - voudraient épargner une contamination à leur partenaire. Elles ne veulent pas que leur partenaire, ou ses enfants, subissent le même sort. Nous devons donc, en tant que médecins, aider les personnes séropositives à rendre leur vie digne d'être vécue et à conserver leur humanité malgré 
un tragique coup du sort. La recommandation de la CFPS concernant le conseil est contraire à l'éthique médicale.

\section{Ne changeons pas la jurisprudence}

La CFPS demande une modification de la jurisprudence pour «toutes les personnes séropositives sous TAR efficace» parce qu'elles ne seraient pas contagieuses: «Un rapport sexuel non protégé d'une personne séropositive sous TAR efficace avec une personne séronégative ne peut être constitutive ni d'une tentative de propagation d'une maladie dangereuse au sens de l'art. 131 du Code pénal suisse ni d'une tentative de lésion corporelle grave selon les art. 122, 123 et 125.»

Comme nous l'avons signalé plus haut, dans l'état actuel de la science, il n'est absolument pas certain qu'une personne séropositive sous TAR efficace ne soit pas sexuellement contagieuse.

En outre, si une telle personne ne vit pas une relation de couple fidèle, elle ne comprendra pas pourquoi elle devrait informer son/sa partenaire sexuel(le) de sa séropositivité et pourquoi elle devrait utiliser le préservatif. Et que vont penser, comment vont se comporter ceux qui réagissent de manière insuffisante au traitement ou qui ne prennent pas leurs médicaments correctement? Et ceux qui ne sont pas encore sous TAR parce que leurs CD4 se situent un peu au-dessus de $350 \mathrm{~mm}^{3}$, ce qui représente une faible charge virale? On sait qu'en matière de sexualité notamment, de nombreuses personnes voient les choses en fonction de leurs désirs.

Par conséquent, pour SIS, il est très important de conserver l'actuelle jurisprudence et d'y rendre attentive la population. Avec sa recommandation, le CFPS mine l'effet préventif de la loi.

\section{Mauvais signal de santé publique adressé à la population}

On s'est rendu compte que les messages des 25 dernières années concernant le safe sex étaient insuffisants, si bien qu'il faut recourir à des stratégies nouvelles et efficaces [20]. Les dernières recommandations de la CFPS n'en font pas partie. Elles laissent penser que le danger de l'infection à VIH est passé, et cela dans une décennie où le taux de nouvelles infections ne recule plus et où l'on observe même dans certains groupes de population une aggravation de l'épidémie [21, 22]. Il y a lieu de s'inquiéter du résultat des recherches qui prouvent qu'en Suisse [23], comme dans d'autres pays industrialisés [24], 30 à 50\% des séropositifs n'apprennent que peu de temps avant l'apparition des symptômes du sida qu'ils sont contaminés et qu'ils transmettent leur in- fection sans le savoir. Il faut considérer ces faits comme un échec de la santé publique [20].

En outre, le seul message de prévention envoyé à la population par la CFPS dans son article est le suivant: pas de pénétration sans préservatif, ni sperme ni sang dans la bouche. Ce message reflète la philosophie préventive des années postmodernes 1980 lorsque l'on pensait que même une infection mortelle ne devait pas restreindre les libertés individuelles. Or depuis lors, le concept de prévention a fondamentalement changé: le préservatif n'est qu'un élément d'une approche globale [25] qui comprend le dépistage, le conseil, un TAR précoce pour les séropositifs, la fidélité dans le couple, le choix minutieux $\mathrm{du} / \mathrm{de}$ la partenaire, l'abstinence, l'information $\mathrm{du} / \mathrm{de}$ la partenaire, la prophylaxie postexposition, etc.

\section{Autres questions}

Les spécialistes se demandent pourquoi la CFPS publie des recommandations qui choquent toute la communauté des experts de la question. Dans une interview accordée à Ars Medici du 14 mars 2008 [26], le professeur Vernazza, président de la CFPS, explique pourquoi cette dernière a publié son article: «Nous voulons lancer un large débat afin d'encourager la déstigmatisation des séropositifs» et, en même temps, inciter à une modification de la jurisprudence afin que les rapports sexuels non protégés de personnes séropositives ne soient plus un délit. Pour ces dernières, les recommandations de la CFPS représentent un soulagement et les libère du stigmate d'être une source de contamination dangereuse pour autrui.

La CFPS a-t-elle perdu tout sens de la mesure? Est-ce pour cette raison qu'elle s'est empêtrée dans les contradictions suivantes? a) Ses recommandations concernant les rapports sexuels sont différentes pour les séropositifs sous TAR efficace vivant une relation de couple fidèle et ceux ne vivant pas une relation de couple fidèle. Mais s'agissant de la jurisprudence, elles ne font pas la différence. b) Dans l'interview, le professeur Vernazza reconnaît que des cas de transmission $\mathrm{du}$ virus sont possibles sous TAR efficace mais dans l'article de la CFPS, sous les intertitres «Importance pour la jurisprudence» et «Importance pour la prévention du sida», on peut lire que «les personnes séropositives qui suivent un TAR efficace ne transmettent pas le VIH par voie sexuelle». c) Pour montrer que les rapports vaginaux d'une personne séropositive sous TAR représentent un risque négligeable, la CFPS, dans son article, les met en parallèle avec les baisers mais elle ne fait même pas la distinction entre 
baisers profonds et baisers sur la joue. Dans l'interview, le professeur Vernazza affirme que baisers et rapports oraux représentent des risques comparables. Or les spécialistes considèrent les rapports oraux (au cours desquels des sécrétions génitales atteignent la muqueuse buccale) comme une voie de transmission sexuelle présentant un risque (certes moindre que les rapports vaginaux).

Dans l'interview, le professeur Vernazza laisse également entendre que la CFPS est tout à fait consciente de formuler des recommandations différentes de celles du reste du monde. Or le virus IH ne connaît aucune frontière.

SIS estime que la population a droit à toute la vérité et n'a pas besoin d'être ménagée par des informations édulcorées. Les recommandations en vue de la prévention du VIH ne doivent pas obéir à des motivations idéologiques et politiques.

La CFPS se trompe quand elle croit que ses recommandations vont mettre un terme à la stigmatisation. Il est évident pour nous médecins qu'il faut s'opposer résolument à toute stigmatisation et discrimination, mais des messages différents provenant des médecins d'une part et des autorités d'autre part désorientent la population et encouragent les comportements irrationnels comme la discrimination des séropositifs.

\section{Références}

1 WHO Regional Office for Europe. Unpublished survey data on file. Novembre 2007.

2 Bernasconi E, Vernazza PL, Bernasconi A, Hirschel B. HIV transmission after suspension of highly active antiretroviral therapy. J Acquir Immune Defic Syndr. 2001;27:209.

3 Castilla J, Del Romero J, Hernando V, Marincovich B, Garcia S, Rodriguez C. Effectiveness of highly active antiretroviral therapy in reducing heterosexual transmission of HIV. J Acquir Immune Defic Syndr. 2005;40:96-101.

4 Vernazza P, Hirschel B, Bernasconi E, Flepp M. Les personnes séropositives ne souffrant d'aucune autre MST et suivant un traitement antirétroviral efficace ne transmettent pas le VIH par voie sexuelle. Bull Méd Suisses. 2008;89(5):165-9.

5 Konsequent Therapierte sind nicht infektiös. NZZ du 31.1.2008, S.15.

6 Sex ohne Kondom für gewisse HIV-Infizierte Schweizer Einschätzung gerät in die Kritik. SDA du 1.2.2008, S.16.

7 Prise de position des autorités sanitaires américaines du 1/2/2008. www.cdc.gov/hiv/resources/press/ 020108.htm.

8 Einschätzung zu HIV-Infizierten in der Kritik. NZZ du 2.2.2008.

9 Neely MN, Benning L, Xu J, et al. Cervical shedding of HIV-1 RNA among women with low levels of viremia while receiving highly active antiretroviral therapy. J Acquir Immune Defic Syndr. 2007;44: $38-42$.
10 Kovacs A, Wasserman SS, Burns D, et al. Determinants of HIV-1 shedding in the genital tract of women. Lancet. 2001;358:1593-601.

11 Cohen MS, Hoffman IF, Royce RA, et al. Reduction of concentration of HIV-1 in semen after treatmen of urethritis: implications for prevention of sexual transmission of HIV-1. Lancet. 1997;349:1868-73.

12 Sadiq ST, Taylor S, Kaye S, et al. The effects of antiretroviral therapy on HIV-1 RNA loads in seminal plasma in HIV-positive patients with and without urethritis. AIDS. 2002;16:219-25.

13 Garcia PM, Kalish LA, Pitt J, et al. Maternal levels of plasma human immunodeficiency virus type 1 RNA and the risk of perinatal transmission. $\mathrm{N}$ Eng J Med. 1999;431:394-402.

14 Rousseau C, Nduati R, Richardson B, et al. Longitudinal analysis of human immunodeficiency virustype 1 RNA in breast milk and of its relationship to infant infection and maternal disease. J Infect Dis. 2003;187:741-7.

15 Barreiro P, del Romero J, Leal M, et al. Natural pregnancies in HIV-serodiscordant couples receiving successful antiretroviral therapy. J Acquir Immune Defic Syndr. 2006;43:324-6.

16 Magder L, Mofenson L, Paul M, et al. Risk faktors and intrapartum transmission of HIV. J Acquir Immune Defic Syndr. 2005;38:87-95.

17 Cane P, Christie I, Dunn D, et al. Time trends in primary HIV drug resistance in the United Kingdom: observational study. BMJ. 2005; 331:1368.

18 Kaplan HS. The Sexual Desire Disorders: Dysfunctional Regulation of Sexual Motivation. Levittown, PA: Brunner/Mazel; 1995.

19 Kaplan HS. The Real Truth About Women and AIDS: How to Eliminate the Risks Without Giving Up Love and Sex. New York, NY: Simon \& Schuster; 1988.

20 Pillay D, Fisher M. Primary HIV infection, phylogenetics, and antiretroviral prevention. JID. 2007; 195:924-6.

21 Office fédéral de la santé publique. L'épidémie de VIH en Suisse 2007. Bulletin de l'OFSP. 2008;28: 84-8.

22 Murphy G, Chartlett A, Brown AE, Gill ON, Parry JV. Is HIV incidence increasing in homo/bisexual men attending GUM clinics in England, Wales and Northern Ireland? Commun Dis Public Health. 2004;7:11-4.

23 Office fédéral de la santé publique. Pas de nouvelle diminution du nombre de cas de sida malgré l'efficacité des thérapies. Bulletin de l'OFSP. 2005; 28:480-8.

24 CDC. Revised Recommendations for HIV Testing of Adults, Adolescents, and Pregnant Women in Health-Care Settings. MMWR. 2006;55(RR14);1-17.

25 Halperin DT, Steiner MJ, Cassell MM, et al. The time has come for common ground on preventing sexual transmission of HIV. Lancet. 2004;364:1913-5.

26 Ferber T. «Wir sind verpflichtet, unseren Patienten das ganze Wissen zu vermitteln.» Ein Gespräch mit dem Infektiologen und Aidsspezialisten Pietro Vernazza. Ars Medici. 2008;98(6):230-1. 